\title{
Gel Dosimetry
}

National Cancer Institute

\section{Source}

National Cancer Institute. Gel Dosimetry. NCI Thesaurus. Code C16184.

Gel dosimetry involves filling a model of a body part, such as the head, neck or breast, with a gel mixture. The gel-filled model (phantom) then undergoes MRI, CT, ultrasound, or radiation therapy, to gauge how effective the same procedure might be for the patient. Radiation alters the chemical structure of the gel, and the changes appear when scientists view a cross-section of the phantom on the computer screen. (from Medscape) 